\title{
Medium access control protocol design for wireless communications and networks review
}

\author{
Zaid Hashim Jaber ${ }^{1,2}$, Dheyaa Jasim Kadhim ${ }^{3}$, Ahmed Sabah Al-Araji ${ }^{1}$ \\ ${ }^{1}$ Department of Computer Engineering, University of Technology, Baghdad, Iraq \\ ${ }^{2}$ Department of Computer Science, College of Science, University of Baghdad, Baghdad, Iraq \\ ${ }^{3}$ Department of Electrical Engineering, College of Engineering, University of Baghdad, Baghdad, Iraq
}

\begin{tabular}{l}
\hline Article Info \\
\hline Article history: \\
Received Apr 29, 2021 \\
Revised Sep 20, 2021 \\
Accepted Oct 9, 2021 \\
\hline
\end{tabular}

Keywords:

Energy efficiency

Medium access control

Massive MIMO

PD-NOMA

Spectral efficiency

Wireless communications and networks

\begin{abstract}
Medium access control (MAC) protocol design plays a crucial role to increase the performance of wireless communications and networks. The channel access mechanism is provided by MAC layer to share the medium by multiple stations. Different types of wireless networks have different design requirements such as throughput, delay, power consumption, fairness, reliability, and network density, therefore, MAC protocol for these networks must satisfy their requirements. In this work, we proposed two multiplexing methods for modern wireless networks: Massive multiple-input-multipleoutput (MIMO) and power domain non-orthogonal multiple access (PD-NOMA). The first research method namely Massive MIMO uses a massive number of antenna elements to improve both spectral efficiency and energy efficiency. On the other hand, the second research method (PD-NOMA) allows multiple non-orthogonal signals to share the same orthogonal resources by allocating different power level for each station. PD-NOMA has a better spectral efficiency over the orthogonal multiple access methods. A review of previous works regarding the MAC design for different wireless networks is classified based on different categories. The main contribution of this research work is to show the importance of the MAC design with added optimal functionalities to improve the spectral and energy efficiencies of the wireless networks.
\end{abstract}

This is an open access article under the CC BY-SA license.

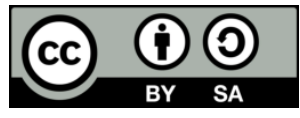

\section{Corresponding Author:}

Zaid Hashim Jaber

Department of Computer Engineering, University of Technology

Al-Wehda neighborhood, Baghdad, Iraq

Email: ce.19.18@grad.uotechnology.edu.iq

\section{INTRODUCTION}

Wireless communications and networks have increasing impact in our recent and future life and are involved in many fields including home networks, cellular networks, the internet of things (IoT) [1]-[3], cloud computing [4]-[6], augment reality (AR) [7], [8], multimedia [9], [10], machine-to-machine (M2M) [11]-[13], and sensors networks [14], [15]. Different wireless networks have different requirements and demands like high throughput, low latency, high reliability, massive connectivity, and energy efficiency. The physical and medium access control (MAC) layers of the wireless networks must be well-designed to satisfy these requirements. Besides, it is better to find a unified solution so various wireless standards like wireless local area network (WLAN) and cellular networks can coexist together in the future [16]-[18].

One of the main problems that degrades the performance of a wireless network is collisions while using a shared or broadcast link with multiple stations trying to access it in a random fashion. Collisions cause interference, data corruption and data retransmission which decline the reliability. More stations mean more 
collisions which make the wireless network non-scalable as well. One of the medium access control (MAC)'s functionalities is handling the shared medium access. In WLAN using distributed coordinated function (DCF) for example, MAC employs carrier sense multiple access/collision avoidance (CSMA/CA) to avoid collisions. CSMA/CA has many components and strategies like exponential backoff timer, interframe spacing, request to send (RTS)/clear to send (CTS) and network allocation vector (NAV) [19], [20]. Much research was proposed to improve CSMA/CA and backoff timer [21]-[23]. Even with collision avoidance, shared medium means less throughput and more latency per station while increasing the number of stations. Another technique that improves medium access for multiple stations is channelization which includes Time-division multiple access (TDMA), frequency division multiple access (FDMA) and code division multiple access (CDMA) with different stations that can share bandwidth using different time, frequency, or code. Two multiplexing techniques that have significantly received big attentions in recent years are massive multiple-input-multipleoutput (massive MIMO) [24]-[26] and power-domain non-orthogonal multiple access (PD-NOMA) [27], [28].

Massive MIMO is a spatial multiplexing method that uses large number of antenna components. It is considered massive if the number of antenna components exceed 64. Massive MIMO improves both spectral and energy efficiencies. It improves spectral efficiency by using different antennas to send and receive different data while it improves energy efficiency by forming beams and focusing their energy into the desired directions [29]. PD-NOMA is a multiplexing technique that improves the spectral efficiency, decreases the latency, and increases the number of active stations by allowing multiple stations to share the same medium resources (frequency, time, and space). PD-NOMA allocates different power to different stations.

One solution to obtain a robust design of modern wireless network is by proposing a new MAC model of the wireless network that consists of multiple cells with massive MIMO that have many medium-contented Orthogonal multiple access (OMA) and non-orthogonal multiple access (NOMA) stations. This model must enhance both the spectral and energy efficiencies, improving throughput and latency per station, allow simultaneous access by multiple stations, have better fairness among stations and finally improve power allocation and management for all stations including base station (BS) and user equipment (UE). The motivation of this paper is to discuss a MAC design for the modern wireless communications and networks by reviewing previous works that involve MAC design of different types of wireless networks based on different categories to improve the spectral efficiency, energy efficiency or both.

Section 2 presents some MAC design principles and concepts. Section 3 explains massive MIMO fundamentals and its advantages. Section 4 explains PD-NOMA as a powerful technology in the wireless communications and networks. Section 5 discusses some previous related works which is categorized based on wireless MAC. Finally, section 6 is the conclusion of this work.

\section{MAC DESIGN PROCEDURE}

MAC sublayer is a part of data link layer within the open system interconnection (OSI) and TCP/IP models as shown in Figures 1(a) and 1(b). One of its main responsibilities is to implement the control mechanism that allows multiple stations to access the medium so they can transmit data. Medium sharing techniques can be categorized to be either static or dynamic [20], [30], [31] as shown in Figure 2 [31]. In static or channelization method, each station is assigned with a static or fixed resource to access the medium like time slot (TDMA), frequency (FDMA) or code (CDMA). On the other hand, dynamic access method, can be either scheduled or random [20], [30], [31]. In scheduled access medium, each station must be authorized to access the medium by other stations before sending the data. So, scheduled method is contention-free. Some of scheduled medium access techniques include: polling and token passing [20], [30], [31]. Finally, random access medium is contention-based method and there is no control over accessing the medium, random-access method is good for medium for medium utilization but its biggest problem is collisions [20], [30], [31].

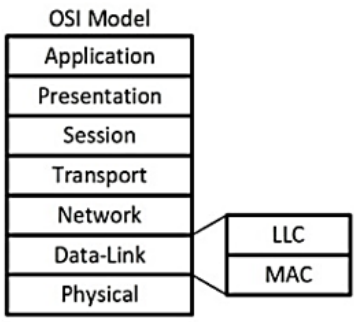

(a)

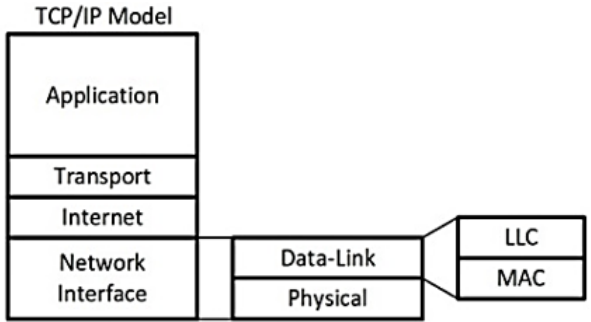

(b)

Figure 1. MAC sublayer within (a) OSI model and (b) TCP/IP model 


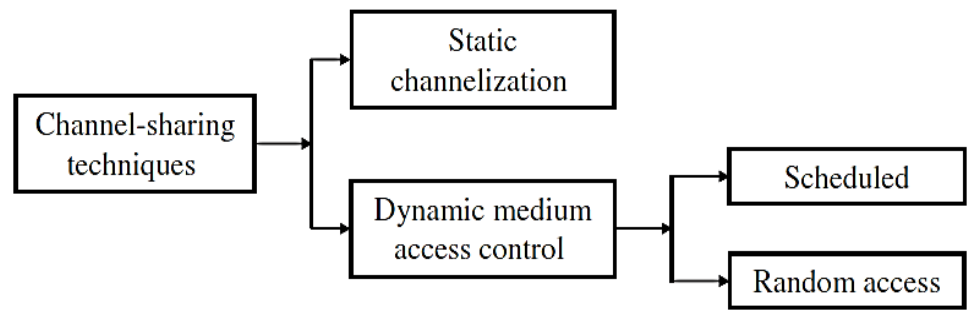

Figure 2. Medium access techniques [31]

\subsection{Random access protocols}

Random access is a medium access method when multiple stations are connected via a broadcast or share medium and there is no priority of one station over the others to access the medium. Random access method is contention-based and not scheduled which requires any station that can access the medium at any time without any central control. One of the biggest problems using random access methods is collision when multiple stations try to send data at the same time which causes conflict and data corruption.

One of the oldest random-access protocols is pure ALOHA [20], [30], [31]. In pure ALOHA, any station can send data whenever it has data to send, this means high probability of collisions. The throughput of pure ALOHA is $\mathrm{S}=\mathrm{Ge}-2 \mathrm{G}$ where $\mathrm{G}$ is the average number of frames generated in one frame time. The maximum throughput of pure ALOHA is 0.184 or $18.4 \%$ successful received frames when $\mathrm{G}=0.5$ [20], [30]. Slotted ALOHA [20], [30], [31] is another random-access protocol that divides time into slots and each time slot represent one frame. All stations must be synchronized to send data at the beginning of these time slots. The throughput of slotted ALOHA is given by $\mathrm{S}=\mathrm{Ge}-\mathrm{G}$. The maximum throughput of slotted ALOHA is 0.368 or $36.8 \%$ successful received frames when $\mathrm{G}=1$, which is better than pure ALOHA [20], [30]. Figure 3 shows the throughput versus the load of both ALOHA methods [30]. Both versions of ALOHA have low medium utilization because of collisions [30]. To minimize the collisions, the stations must listen to the medium before trying to send data to check if the medium is idle or busy. This method is called CSMA [20], [30], [31] and it is based on listen-before-talk (LBT) technique. CSMA has many persistence methods which define behavior when stations find the medium busy (continuous sense or wait). Figure 4 shows the thought versus load comparison for different random-access protocols [30]. To increase the performance of CSMA, other two variations were added to CSMA. The first one is CSMA with collision detection (CSMA/CD) which is the basic Ethernet MAC protocol, and CSMA with collision avoidance (CSMA/CA) which used in 802.11 WLAN.

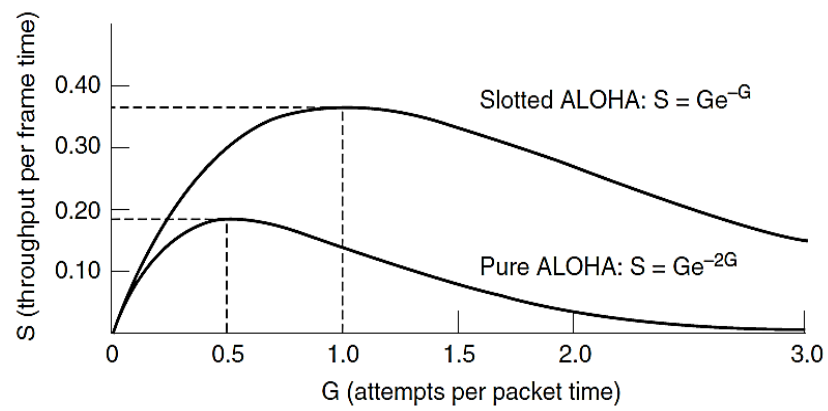

Figure 3. Throughput versus load for ALOHA systems [30]

\subsection{IEEE 802.11 MAC sublayer}

In IEEE 802.11 WLAN, the MAC architecture can be represented in many operation modes as shown in Figures 5 [32]. DCF is a random and contention-based access method with no central control, while point coordination function (PCF) is a central and contention-free access method using polling. Also, hybridcoordination function (HCF) is a combination of both DCF and PCF and it is mainly used with quality of service (QoS) implementation. DCF is the fundamental access method in wireless LAN and PCF is optional method built on the top of DCF. For QoS purposes in IEEE 802.11e, extended DCF or enhanced DCF (EDCF) [33] is used to prioritize the different types of traffic. The EDCF is a HFC that uses polling mechanism with contention-based and contention free access methods with multiple priorities. 
DCF uses CSMA/CA to access the medium with a binary backoff timer to reduce the collisions in busy medium, collision avoidance (CA) is used instead of collision detection (CD) because wireless radios are half-duplex so they cannot send data and detect collisions at the same time, besides the transmitted signal is according to the transmitter perspective is much stronger than any other signals in the medium [19], [30]. CSMA/CA is implemented through many components including: physical and virtual carrier sensing, interframe spacing (IFS), contention window (CW) with binary backoff timer and positive acknowledgment [19], [20]. To reduce the collisions, IFS is used to defer data transmission in the medium even when if it is idle i.e., IFS is the time interval between different types of frames [19], [20], [32]. Figure 6 shows six types of IFS which are from shortest to largest: reduced interframe space (RIFS), short interframe space (SIFS), PCF interframe space (PIFS), DCF interframe space (DIFS), arbitration interframe space (AIFS) and extended interframe space (EIFS) [32]. These different types of IFS allow for prioritization of different types of frames for example ACK has more priority than actual data and has a chance to go first to the medium because ACK waits SIFS period while actual data follows at least DIFS period assuming zero backoff timer.

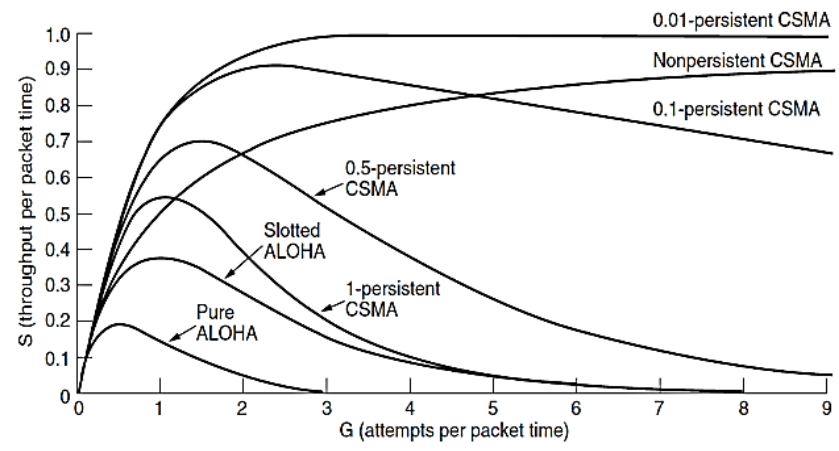

Figure 4. Thought versus load comparison for different random-access protocols [30]

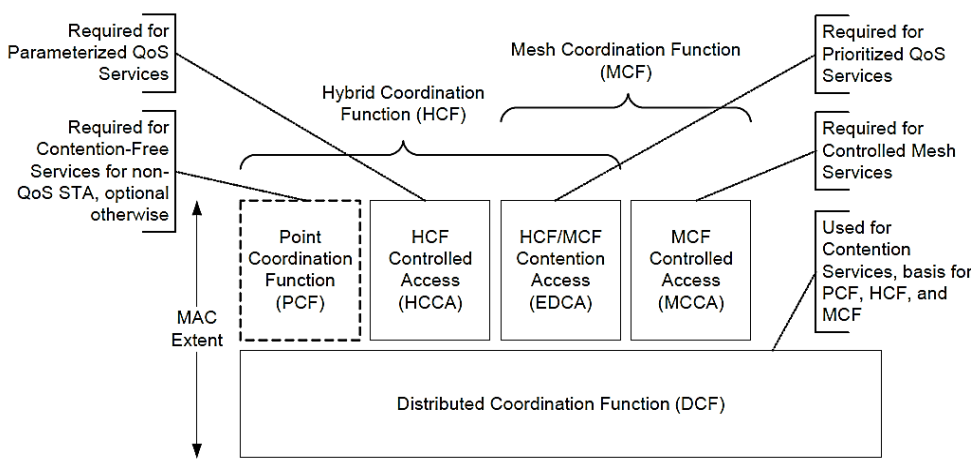

Figure 5. IEEE 802.11 MAC architecture [32]

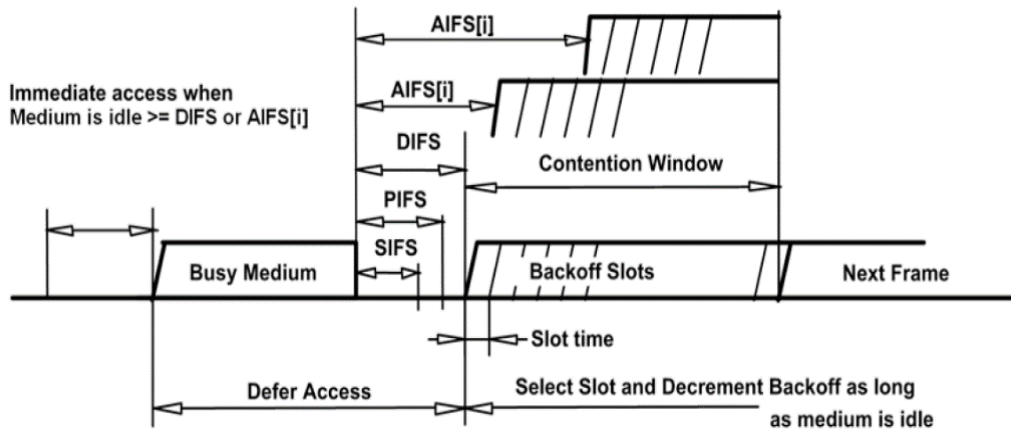

Figure 6. IFS types [32] 
Both physical and virtual customer service (CS) are implemented in discounted cash flow (DCF) [19], [20], [30], [32]. In physical CS, the station must listen to medium prior sending data to check if it is busy or not. In virtual CS, the station reserves the medium through the request to send (RTS) and clear to send (CTS) exchange before sending any data. Duration field of the RTS and CTS frames represents the time required to send the actual data and return ACK back, other stations must update their NAV timer to the received RTS or/and CTS with valid duration ID field. NAV timer is the time required by stations to pass before checking the idleness of the medium. Figure 7 shows the NAV setting when receiving RTS and/or CTS frames [32], note that NAV setting requires either receiving RTS or CTS frame at least which solves the case of hidden node problem.

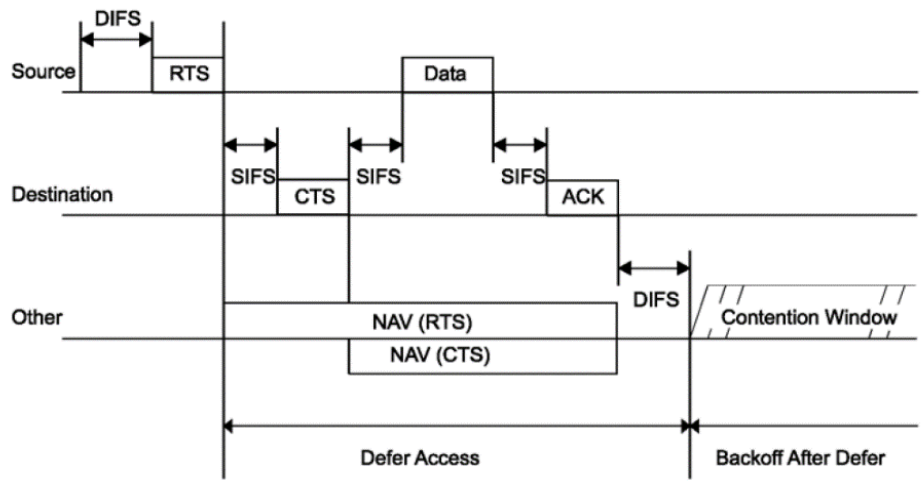

Figure 7. NAV setting [32]

To send data frame, the station must use CS to determine whether the medium is idle or busy. If the medium is busy, the station must wait until the medium becomes idle without any interruption for DIFS period. After this DIFS period, the station must wait another period called backoff period before sending data. The random backoff time is given by [32]:

$$
\text { Backoff Time }=\text { Random }() \times \text { aSlotTime }
$$

where Random () is an integer value and it is uniformly distributed over period $[0, \mathrm{CW}]$ where $\mathrm{CW}$ or contention window is another integer value within the range CWmin and CWmax which are determined by PHY characteristics and aSlotTime is the slot period and determined by PHY characteristics. The purpose of random exponential backoff time is to reduce the collisions during the contention between multiple stations trying to access the medium at the same time. Under the condition of many stations with heavy load, each attempt for data retransmission after collision leads to exponential increase in $\mathrm{CW}$ and backoff timer but it never exceeds CWmax value as shown in Figure 8 [32].

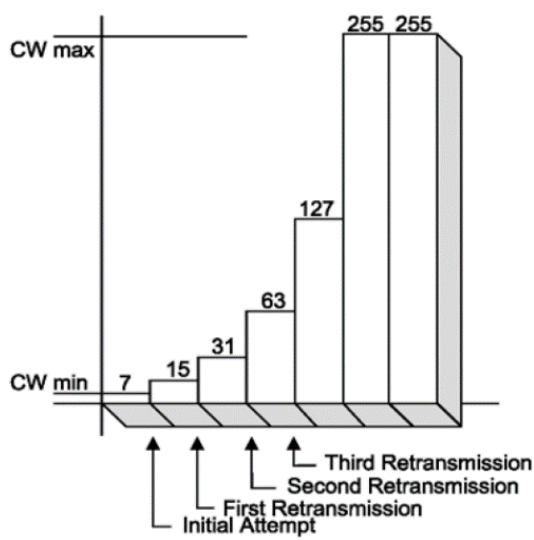

Figure 8. Exponential increase in CW [32] 
CSMA/CA with all previously mentioned techniques reduce the probability of the collisions but do not prevent it. Positive ACK is used as a verification method of the data delivery. If the data is corrupted and delivered correctly to the intended because of the collisions, then the sender radio will not receive ACK from the receiver radio. As a result, the data must be retransmitted again. To detect the overlapping basic service set (OBSS) co-channel interference, BSS coloring is defined in the PHY and MAC layers of IEEE 802.11ax to identify the frames with the same color as interference. This technique can be used for spatial reuse [34], [35].

\subsection{Adaptive MAC for smart antenna}

Smart antenna is used to increase throughputs in ad hoc wireless networks. This is done by implementing direction of arrival (DOA) algorithms [36] to help to determine the directions of beams and nulls. The antenna of the sender can form a beam directed to the receiver antenna and vice versa. Also, the receiver can determine the direction of multiple senders at the same time which increases throughputs. Nulls can be placed in the direction of interferences to minimize their effects [33]. MAC layer design can be added to these smart antennas to control the directions of beams and nulls. Smart-ALOHA [37] and Smart-802b [38] are proposed protocols for smart antennas which are a modified version of ALOHA and 802.11b. These modified protocols use the properties of smart antennas: beamforming, nulling and DOA in their work.

\section{FIRST PROPOSED RESEARCH METHOD: MASSIVE MIMO}

MIMO is a technique used to increase the spectral efficiency and improve the performance of wireless communication through using multiple transmission and receiving antennas to exploit the multipath of wireless signal. MIMO improves the average channel capacity for both non-fading additive white Gaussian noise (AWGN) channel and Rayleigh fading channel [39]. MIMO is widely used in recent wireless communications standards like IEEE $802.11 \mathrm{n}$ and IEEE $802.11 \mathrm{ac}$ Wi-Fi and $3 \mathrm{G}$ and beyond cellular networks.

MIMO can be found in many forms, the simplest one is point-to-point MIMO [40], [41] which is represented by a single BS with $M$ antennas that serves a single UE with $k$ antennas. Point-to-point MIMO can be used to serve multiple UEs, but they must be orthogonally multiplexed in time and/or frequency. Another form of MIMO is the multi-user MIMO (MU-MIMO) [40], [41] which consists of single BS with $M$ antennas that serves $k$ number of UEs each has a single antenna (or more) using the same frequency-time resources which increases the spectral efficiency compared to the point-to-point MIMO.

Massive MIMO [40], [41] is the ultimate version of MU-MIMO and it is more scalable with much greater number of BS antennas, and the number of BS antenna is larger than the number of UEs served. In massive MIMO, each BS may have hundreds or thousands number of antennas that can serve tens or hundreds of UEs using the same frequency resources. When massive MIMO operated in time division duplex (TDD) and exploits reciprocity of the channel, the resources required for pilots depends only on the number of active UEs. So only BTS must have CSI and no channel estimation is required at UEs because of channel hardening. Massive MIMO is very scalable when increasing the number of BS antennas and provides nearly optimal linear processing at BS.

Massive MIMO is one of the main technologies that are proposed for $5 \mathrm{G}$ and beyond. Massive number of antennas can provide two advantages. First, it improves the energy efficiency by focusing the energy of the wireless signal in the desired directions through narrow beams and nulling the signals in the undesired directions. Second, it improves the spectral efficiency by allowing transmission and receiving multiple independent signals using the same frequency and time resources. Figure 9 shows a schematic diagram of a single cell massive MIMO system [29].

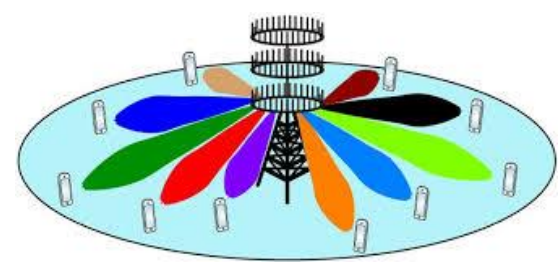

Figure 9. Single cell massive MIMO [29]

In general, massive MIMO enhances the performance of the wireless communications and networks by reducing the multipath effects in the fading channels and forming beams toward the desired directions. Besides, massive MIMO has better link quality than the conventional MU-MIMO in a frequency-selective and 
small-scale fading channels because of channel hardening [42]. In MIMO technologies, there are many decoding and precoding schemes to support multiple uplink stream from UEs to BS and multiple downlink streams from BS to UEs [43]. Multi-user detection (MUD) schemes are used for simultaneous uplink receptions while multi-user interference cancellation (MUIC) schemes are used for simultaneous downlink transmissions. MAC design can be extended to be involved in many aspects like decoding/precoding schemes, channel state information (CSI) acquisitions and scheduling schemes [43].

\section{SECOND PROPOSED RESEARCH METHOD: PD-NOMA}

In wireless communications, the number of active users is limited to the available orthogonal resources such as different time slots in TDMA and different frequency channels in FDMA to avoid interference. NOMA is a promising technique for $5 \mathrm{G}$ systems and beyond to allow multiple users to share the same orthogonal resources and thus increase the overall throughput and improve spectral efficiency. NOMA can be implemented mainly either in the power domain or in the code domain [29].

The main advantages of NOMA are massive connectivity, lower latency, and better spectral efficiency. Unlike OMA, NOMA is not limited to the orthogonal resources and this means that the number of active users is theoretically unlimited. Sharing the same resource by multiple users in NOMA leads to lower access time and higher data rate also [29], [44]. In PD-NOMA, different NOMA users can share the same spectral band at the same time but with different power allocated to them [29], [44]. Figure 10 the difference between OMA and PD-NOMA systems and both spectral and power allocations [29].
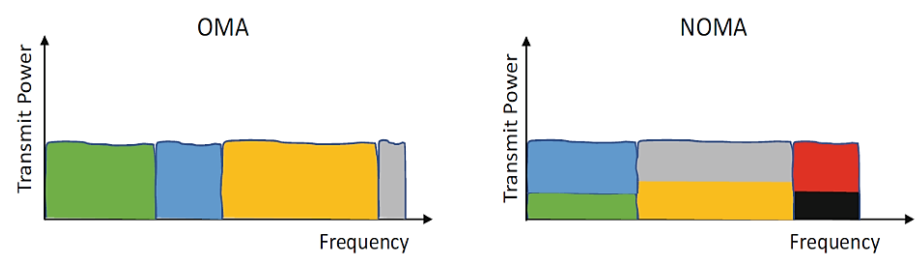

Figure 10. Spectral and power allocations for OMA and NOMA [29]

The direction of the wireless communications can be either downlink or uplink. The downlink or broadcast channel (BC) is used for data transmission from the BS to the UEs while the uplink or medium access channel (MAC) is used for data transmission from the UEs to the BS. The BS decodes the data using the superposition coding (SC) for power domain multiplexing before sending it via the BC. To decode the data on the other end, the near user (with high channel gain) uses successive interference cancellation (SIC) to extract its signal from the far user signal, while the far user (with low channel gain) treats the near user signal as noise. Figure 11 shows power domain multiplexing for two users in a single cell downlink NOMA [29].

In the case of a single cell with two users [29], [45], [46]. For OMA, if user $_{1}$ has $\alpha$ fraction of the resource share and user $_{2}$ has $\bar{\alpha}$ fraction of the resource share where $0 \leq \alpha \leq 1$ and $\bar{\alpha} \triangleq 1-\alpha$, then the data rates for these OMA users in both $\mathrm{BC}$ and MAC are:

$$
\begin{aligned}
& R_{1}=\frac{\alpha}{2} \log _{2}\left(1+\left|h_{1}\right|^{2} P\right) \\
& R_{2}=\frac{\bar{\alpha}}{2} \log _{2}\left(1+\left|h_{2}\right|^{2} P\right)
\end{aligned}
$$

Considering that $\left|h_{n}\right| P$ is the received signal-to-noise ratio (SNR) for the $u s e r_{n}$, where $h_{n}$ is the channel gain, $P$ is the transmitting power and the power of noise is normalized to 1 . The data rates of the MAC can be increased by applying power control (PC) as (4), (5):

$$
\begin{aligned}
& R_{1}=\frac{\alpha}{2} \log _{2}\left(1+\frac{\left|h_{1}\right|^{2} P}{\alpha}\right) \\
& R_{2}=\frac{\bar{\alpha}}{2} \log _{2}\left(1+\frac{\left|h_{2}\right|^{2} P}{\bar{\alpha}}\right)
\end{aligned}
$$

In NOMA, both users send their signals at the same time via the uplink channel or MAC and they interfere with each other. The BS uses SIC to extract these signals. The data rates can be written as (6), (7): 


$$
\begin{aligned}
& R_{1}=\frac{1}{2} \log _{2}\left(1+\left|h_{1}\right|^{2} P\right) \\
& R_{2}=\frac{1}{2} \log _{2}\left(1+\frac{\left|h_{2}\right|^{2} P}{\left|h_{1}\right|^{2} P+1}\right)
\end{aligned}
$$

Figure 12(a) shows that the NOMA is much better capacity region in the MAC than the OMA without power control [29]. For the downlink channel or BC from BS to the users, the BS performs (SC) and the users perform (SIC). If $\beta$ is the $\mathrm{BS}$ power fraction allocated to the user1's data, such that $\bar{\beta} \triangleq 1-\beta$ and $0 \leq \beta \leq 1$. The data rates in the $\mathrm{BC}$ are:

$$
\begin{aligned}
& R_{1}=\frac{1}{2} \log _{2}\left(1+\beta\left|h_{1}\right|^{2} P\right) \\
& R_{2}=\frac{1}{2} \log _{2}\left(1+\frac{\bar{\beta}\left|h_{2}\right|^{2} P}{\beta\left|h_{2}\right|^{2} P+1}\right)
\end{aligned}
$$

The NOMA increase the capacity region in BC as shown in Figure 12(b) [29]. NOMA also improve the user fairness by increasing the power allocation with poor channel gain. NOMA can be combined with MIMO technologies to achieve higher spectral efficiency.

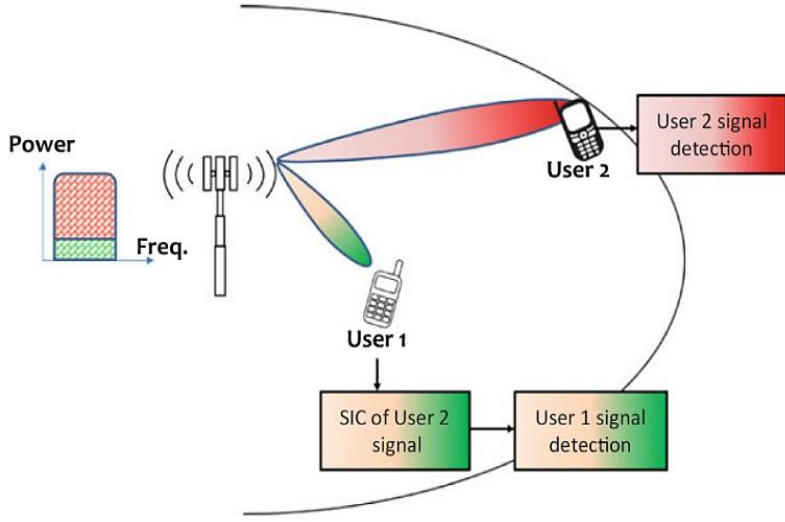

Figure 11. Power domain multiplexing for two users in a single cell downlink NOMA [29]

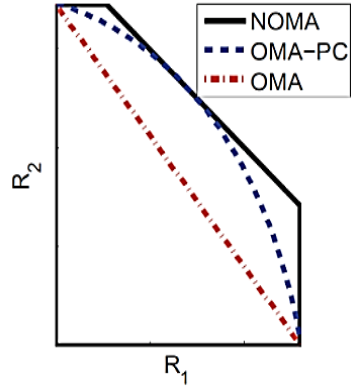

(a)

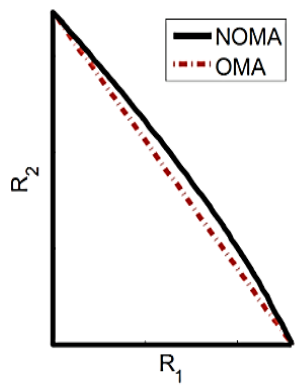

(b)

Figure 12. Data rates of OMA and NOMA for the two-user (a) uplink (MAC) and (b) downlink (BC) in $\mathrm{P}_{1}=5$,

$$
\mathrm{P}_{2}=10 \text { [29] }
$$

\section{REVIEW OF RELEVANT WORKS}

There are many categories that can be used to optimize the MAC design of the wireless communications. These categories can be used to improve the spectral efficiency, energy efficiency or both [47], [48]. Some of previous related works which include MAC design of wireless communications and networks are summarized in Table 1. The main categories for this review are channel utilization, fairness, 
availability, QoS, power saving and efficiency of resource allocation [49]-[53]. This survey involves the MAC design for the different types of wireless networks such as 802.11 WLAN, 5G and beyond cellular networks, sensor networks, body networks and other.

Table 1. Review of MAC design of wireless communications and networks

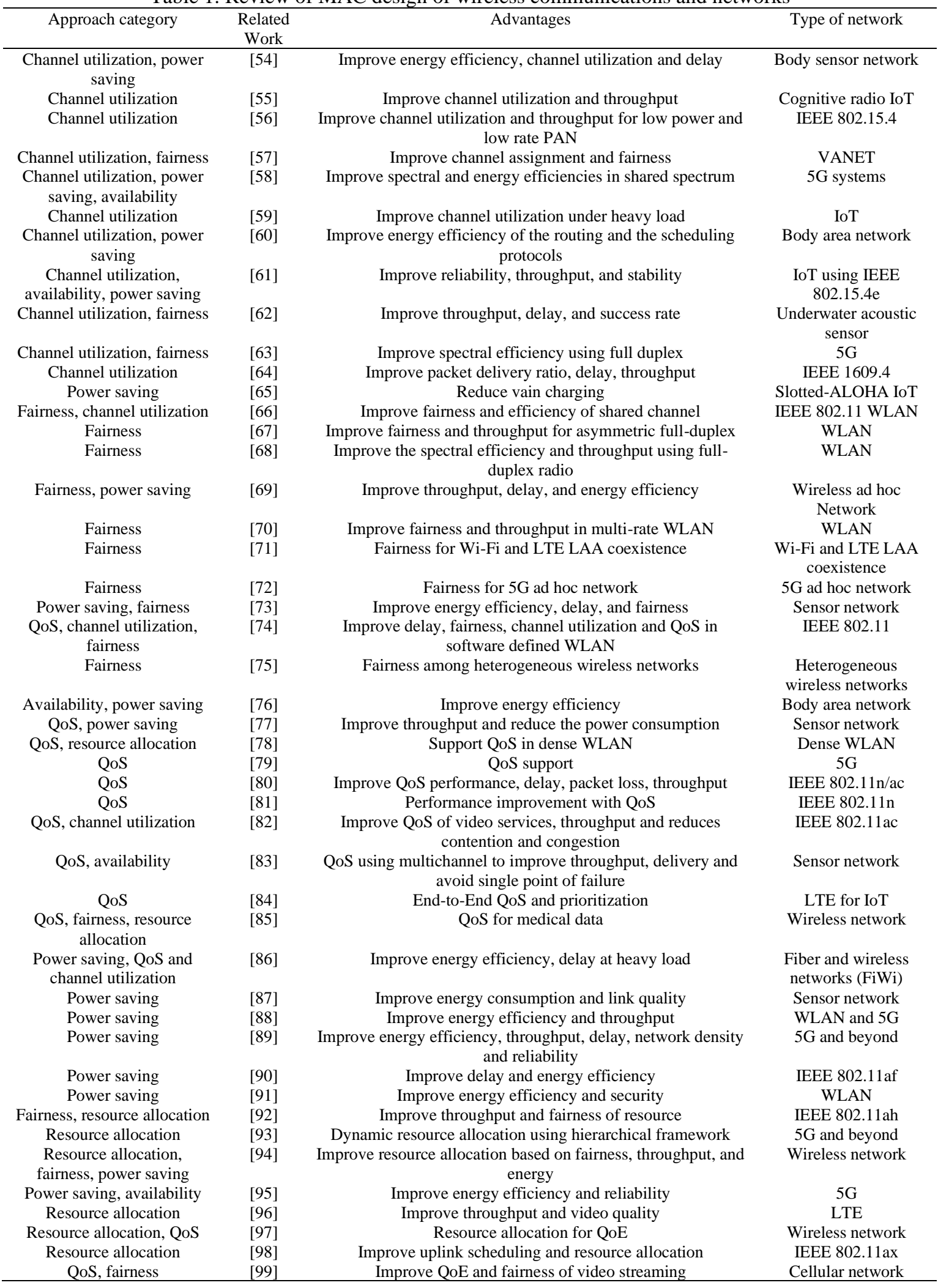




\section{CONCLUSION}

The main contribution of this work is to discuss the MAC layer of the wireless networks as the main part in the multiplexing process of the modern wireless networks. Massive MIMO and PD-NOMA are discussed as important multiplexing techniques to increase the spectral efficiency in wireless communications. A review is presented for MAC design of different wireless communications and networks and is classified according to different categories. The categories used in this review are channel utilization, fairness, availability, QoS, power saving, and resource allocation.

As mentioned above, the expected contribution for this work to add an optimal functionality at the MAC layer using one or more of the optimization techniques such as: particle swarm optimization (PSO), base algorithm (BA), whale optimization algorithm (WOA), and genetic algorithm (GA). We can use these optimization algorithms to directly control the antennas as well as control the direction of the beams and the direction of the nulls, then this approach may show a very high throughput while maintaining the fairness which it is the main MAC function. Besides, we can improve the power management and allocation for NOMA stations to increase throughput and guarantee a better fairness. Consequently, we can optimize the backoff timer when increasing the number of users to reduce latency and improve fairness. Also, we can minimize the energy consumption of the mobile users without any negative impacts on throughput, latency, and fairness.

\section{REFERENCES}

[1] L. Chettri and R. Bera, “A comprehensive survey on internet of things (IoT) toward 5G wireless systems,” IEEE Internet of Things Journal, vol. 7, no. 1, pp. 16-32, Jan. 2020, doi: 10.1109/JIOT.2019.2948888.

[2] J. Ding, M. Nemati, C. Ranaweera, and J. Choi, "IoT connectivity technologies and applications: A survey," IEEE Access, vol. 8, pp. 67646-67673, 2020, doi: 10.1109/ACCESS.2020.2985932.

[3] A. Cilfone, L. Davoli, L. Belli, and G. Ferrari, "Wireless mesh networking: An IoT-oriented perspective survey on relevant technologies,” Future Internet, vol. 11, no. 4, Art. no. 99, Apr. 2019, doi: 10.3390/fi11040099.

[4] Y. Wang, I. R. Chen, and D. C. Wang, "A survey of mobile cloud computing applications: perspectives and challenges," Wireless Personal Communications, vol. 80, no. 4, pp. 1607-1623, Oct. 2015, doi: 10.1007/s11277-014-2102-7.

[5] Y. Cui, X. Ma, H. Wang, I. Stojmenovic, and J. Liu, "A survey of energy efficient wireless transmission and modeling in mobile cloud computing," Mobile Networks and Applications, vol. 18, no. 1, pp. 148-155, Apr. 2013, doi: 10.1007/s11036-012-0370-6.

[6] A. Botta, W. De Donato, V. Persico, and A. Pescapé, "Integration of cloud computing and internet of things: A survey," Future Generation Computer Systems, vol. 56, pp. 684-700, Mar. 2016, doi: 10.1016/j.future.2015.09.021.

[7] Di. Chatzopoulos, C. Bermejo, Z. Huang, and P. Hui, "Mobile augmented reality survey: From where we are to where we go," IEEE Access, vol. 5, pp. 6917-6950, 2017, doi: 10.1109/ACCESS.2017.2698164.

[8] Z. Huang, P. Hui, C. Peylo, and D. Chatzopoulos, "Mobile augmented reality survey: a bottom-up approach," arxiv.org/abs/1309.4413v2, 2013.

[9] A. Sharif, V. Potdar, and E. Chang, "Wireless multimedia sensor network technology: A survey," in IEEE International Conference on Industrial Informatics (INDIN), Jun. 2009, pp. 606-613, doi: 10.1109/INDIN.2009.5195872.

[10] M. Amjad, M. H. Rehmani, and S. Mao, "Wireless multimedia cognitive radio networks: A comprehensive survey," IEEE Communications Surveys and Tutorials, vol. 20, no. 2, pp. 1056-1103, 2018, doi: 10.1109/COMST.2018.2794358.

[11] E. Kartsakli et al., "A survey on M2M systems for mhealth: a wireless communications perspective,” Sensors (Switzerland), vol. 14, no. 10, pp. 18009-18052, Sep. 2014, doi: 10.3390/s141018009.

[12] P. K. Verma et al., "Machine-to-Machine (M2M) communications: A survey," Journal of Network and Computer Applications, vol. 66, pp. 83-105, May 2016, doi: 10.1016/j.jnca.2016.02.016.

[13] P. Rawat, K. D. Singh, and J. M. Bonnin, "Cognitive radio for M2M and internet of things: A survey," Computer Communications, vol. 94, pp. 1-29, Nov. 2016, doi: 10.1016/j.comcom.2016.07.012.

[14] S. M. Mohamed, H. S. Hamza, and I. A. Saroit, "Coverage in mobile wireless sensor networks (M-WSN): A survey," Computer Communications, vol. 110, pp. 133-150, Sep. 2017, doi: 10.1016/j.comcom.2017.06.010.

[15] A. Ali, Y. Ming, S. Chakraborty, and S. Iram, "A comprehensive survey on real-time applications of WSN," Future Internet, vol. 9, no. 4, Art. no. 77, Nov. 2017, doi: 10.3390/fi9040077.

[16] M. Ayyash et al., "Coexistence of WiFi and LiFi toward 5G: Concepts, opportunities, and challenges," IEEE Communications Magazine, vol. 54, no. 2, pp. 64-71, Feb. 2016, doi: 10.1109/MCOM.2016.7402263.

[17] A. Mamadou Mamadou, J. Toussaint, and G. Chalhoub, "Survey on wireless networks coexistence: resource sharing in the 5G Era," Mobile Networks and Applications, vol. 25, no. 5, pp. 1749-1764, Jun. 2020, doi: 10.1007/s11036-020-01564-w.

[18] G. Naik, J. M. Park, J. Ashdown, and W. Lehr, "Next generation Wi-Fi and 5G NR-U in the 6 GHz bands: opportunities and challenges," IEEE Access, vol. 8, pp. 153027-153056, 2020, doi: 10.1109/ACCESS.2020.3016036.

[19] D. D. Coleman and D. A. Westcott, CWNA: Certified wireless network administrator official study guide: Exam CWNA-106, 4th ed. In. USA, 2014.

[20] B. A. Forouzan, Data communications and networking, 5th Edition, NY, USA: McGraw Hill, 2013.

[21] H. Qi, Z. Hu, X. Wen, and Z. Lu, “An enhanced MAC backoff algorithm for heavy user loaded WLANs,” in 2017 IEEE Wireless Communications and Networking Conference (WCNC), Mar. 2017, pp. 1-6, doi: 10.1109/WCNC.2017.7925824.

[22] M. Gamal, N. Sadek, M. R. M. Rizk, and M. A. E. A. Ahmed, "Optimization and modeling of modified unslotted CSMA/CA for wireless sensor networks," Alexandria Engineering Journal, vol. 59, no. 2, pp. 681-691, Apr. 2020, doi: 10.1016/j.aej.2020.01.035.

[23] A. Jamali, S. M. S. Hemami, M. Berenjkoub, and H. Saidi, "An adaptive MAC protocol for wireless LANs," Journal of Communications and Networks, vol. 16, no. 3, pp. 311-321, Jun. 2014, doi: 10.1109/JCN.2014.000052.

[24] L. Lu, G. Y. Li, A. L. Swindlehurst, A. Ashikhmin, and R. Zhang, "An overview of massive MIMO: Benefits and challenges," IEEE Journal on Selected Topics in Signal Processing, vol. 8, no. 5, pp. 742-758, Oct. 2014, doi: 10.1109/JSTSP.2014.2317671.

[25] N. Hassan and X. Fernando, "Massive MIMO wireless networks: An overview," Electronics (Switzerland), vol. 6, no. 3, Art. no. 63, Sep. 2017, doi: 10.3390/electronics6030063.

[26] P. Vardhan, M. Gupta, and A. Kumar, "Massive-MIMO- past, present and future: A review," Indian Journal of Science and 
Technology, vol. 9, no. 48, Dec. 2016, doi: 10.17485/ijst/2016/v9i48/99891.

[27] M. Liaqat, K. A. Noordin, T. Abdul Latef, and K. Dimyati, "Power-domain non orthogonal multiple access (PD-NOMA) in cooperative networks: an overview," Wireless Networks, vol. 26, no. 1, pp. 181-203, Jul. 2020, doi: 10.1007/s11276-018-1807-z.

[28] C. H. Liu and D. C. Liang, "Heterogeneous networks with power-domain NOMA: coverage, throughput, and power allocation analysis," IEEE Transactions on Wireless Communications, vol. 17, no. 5, pp. 3524-3539, May 2018, doi: 10.1109/TWC.2018.2816923.

[29] M. Vaezi, Z. Ding, and H. Vincent Poor, Multiple access techniques for $5 G$ wireless networks and beyond. Springer International Publishing, 2018.

[30] A. S. Tanenbaum and D. (David) Wetherall, Computer networks, 5th ed. Bo. USA: Pearson Prentice Hall, 2011.

[31] D. P. Agrawal and Q.-A. Zeng, Introduction to wireless and mobile systems, 3rd ed. USA: Cengage learning, 2011.

[32] "IEEE Standard for Information technology-Telecommunications and information exchange between systems local and metropolitan area networks-specific requirements-Part 11: wireless LAN medium access control (MAC) and physical layer (PHY) specifications," IEEE Std 802.11-2012 (Revision of IEEE Std 802.11-2007), pp. 1-2793, 2012, doi: 10.1109/IEEESTD.2012.6178212.

[33] S. Glisic and J. P. Mäkelä, "Advanced wireless networks: 4G technologies," in IEEE International Symposium on Spread Spectrum Techniques and Applications, Aug. 2006, pp. 442-446, doi: 10.1109/ISSSTA.2006.311811.

[34] F. Wilhelmi, S. Barrachina-Muñoz, C. Cano, I. Selinis, and B. Bellalta, "Spatial Reuse in IEEE 802.11ax WLANs," Computer Communications, vol. 170, pp. 65-83, Mar. 2021, doi: 10.1016/j.comcom.2021.01.028.

[35] A. Valkanis, A. Iossifides, P. Chatzimisios, M. Angelopoulos, and V. Katos, "IEEE 802.11ax spatial reuse improvement: An interference-based channel-access algorithm," IEEE Vehicular Technology Magazine, vol. 14, no. 2, pp. 78-84, Jun. 2019, doi: 10.1109/MVT.2019.2904101.

[36] H. Singh and S. Singh, "DOA-ALOHA: Slotted ALOHA for ad hoc networking using smart antennas," in IEEE Vehicular Technology Conference, 2003, vol. 58, no. 5, pp. 2804-2808, doi: 10.1109/vetecf.2003.1286112.

[37] H. Singh and S. Singh, "Smart-Aloha for multi-hop wireless networks," Mobile Networks and Applications, vol. 10, no. 5, pp. 651-662, Oct. 2005, doi: 10.1007/s11036-005-3360-0.

[38] H. Singh and S. Singh, "Smart-802.11b MAC protocol for use with smart antennas," in IEEE International Conference on Communications, 2004, vol. 6, pp. 3684-3688, doi: 10.1109/icc.2004.1313230.

[39] P. Varzakas, "Average channel capacity for Rayleigh fading spread spectrum MIMO systems," International Journal of Communication Systems, vol. 19, no. 10, pp. 1081-1087, 2006, doi: 10.1002/dac.784.

[40] T. L. Marzetta, E. G. Larsson, H. Yang, and H. Q. Ngo, Fundamentals of massive MIMO. Cambridge University Press, 2016.

[41] H. Q. Ngo, Massive MIMO: fundamentals and system designs. Linköping University Electronic Press, 2015.

[42] E. Björnson, J. Hoydis, and L. Sanguinetti, "Massive MIMO networks: Spectral, energy, and hardware efficiency," Foundations and Trends in Signal Processing, vol. 11, no. 3-4, pp. 154-655, 2017, doi: 10.1561/2000000093.

[43] R. Liao, B. Bellalta, M. Oliver, and Z. Niu, "MU-MIMO MAC protocols for wireless local area networks: A survey," IEEE Communications Surveys and Tutorials, vol. 18, no. 1, pp. 162-183, 2016, doi: 10.1109/COMST.2014.2377373.

[44] V. Bhatia, P. Swami, S. Sharma, and R. Mitra, Non-orthogonal multiple access: an enabler for massive connectivity, vol. 100, no. 2. Springer International Publishing, 2020.

[45] Y. Saito, Y. Kishiyama, A. Benjebbour, T. Nakamura, A. Li, and K. Higuchi, "Non-orthogonal multiple access (NOMA) for cellular future radio access," in 2013 IEEE 77th Vehicular Technology Conference (VTC Spring), Jun. 2013, pp. 1-5, doi: 10.1109/VTCSpring.2013.6692652.

[46] K. Higuchi and A. Benjebbour, "Non-orthogonal multiple access (NOMA) with successive interference cancellation for future radio access," IEICE Transactions on Communications, vol. E98.B, no. 3, pp. 403-414, 2015, doi: 10.1587/transcom.E98.B.403.

[47] W. Al-Jawher and D. Kadhim, "A proposal algorithm to solve delay constraint least cost optimization problem," Engineering Journal, 2013.

[48] D.J. Kadhim and O.A. Hamad, "Improving IoT applications using a proposed routing protocol," Journal of Engineering, vol. 20, no. 11 , pp. 50-62, 2014.

[49] H. S. A. H.N. Abdullah, "Low energy consumption for cooperative and non-cooperative cognitive radio," Engineering and Technology Journal, vol. 35, no. 3, pp. 222-228, 2017.

[50] S.A. Rafea and A.A. Kadhim, "Routing with energy threshold for WSN-IoT based on RPL protocol," Iraqi Journal of Computer, Communication, Control and System Engineering, pp. 71-81, Feb. 2019, doi: 10.33103/uot.ijccce.19.1.9.

[51] N. S. B. H.N. Abdullah and M. T. S. Al-Kaltakchi, "Proposed two-stage detection rules for improving throughput in cognitive radio networks," Iraqi Journal of Computer, Communication, Control and System Engineering, pp. 1-11, Oct. 2019, doi: 10.33103/uot.ijecce.19.4.1.

[52] I. H. Ali., "Implement wireless transceiver system based on convolutional coding; aided by soft-bit decoding," Iraqi Journal of Computer, Communication, Control and System Engineering, pp. 49-55, May 2018, doi: 10.33103/uot.ijccce.18.1.5.

[53] A. E. Abdelkareem, "Convolutional code constraint length over rayleigh fading channel: performance evaluation and hardware aspects," IIraqi Journal Of Computers, Communications, Control And Systems Engineering, vol. 17, no. 1, pp. 42-48, 2017.

[54] A. K. Pandey and N. Gupta, "An energy efficient distributed queuing random access (EE-DQRA) MAC protocol for wireless body sensor networks," Wireless Networks, vol. 26, no. 4, pp. 2875-2889, Jan. 2020, doi: 10.1007/s11276-019-02244-8.

[55] M. Aloqaily, H. Bany Salameh, I. Al Ridhawi, K. Batieha, and J. Ben Othman, "A multi-stage resource-constrained spectrum access mechanism for cognitive radio IoT networks: Time-spectrum block utilization," Future Generation Computer Systems, vol. 110, pp. 254-266, Sep. 2020, doi: 10.1016/j.future.2020.04.022.

[56] A. Gezer and S. Okdem, "Improving IEEE 802.15.4 channel access performance for IoT and WSN devices," Computers and Electrical Engineering, vol. 87, Art. no. 106745, Oct. 2020, doi: 10.1016/j.compeleceng.2020.106745.

[57] U. Mohanakrishnan and B. Ramakrishnan, "MCTRP: An energy efficient Tree routing protocol for vehicular Ad Hoc network using genetic whale optimization algorithm," Wireless Personal Communications, vol. 110, no. 1, pp. 185-206, Aug. 2020, doi: 10.1007/s11277-019-06720-4.

[58] A. Ercan, M. O. Sunay, and I. F. Akyildiz, "RF energy harvesting and transfer for spectrum sharing cellular IoT communications in 5G systems," IEEE Transactions on Mobile Computing, vol. 17, no. 7, pp. 1680-1694, Jul. 2018, doi: 10.1109/TMC.2017.2740378.

[59] O. Gurewitz and O. Zaharia, "Collision prevention for duty-cycle receiver-initiation mac protocol via multiple access reservation (Mar-rimac)," Sensors (Switzerland), vol. 21, no. 1, pp. 1-30, Dec. 2021, doi: 10.3390/s21010127.

[60] G. Yang, X. W. Wu, Y. Li, and Q. Ye, "Energy efficient protocol for routing and scheduling in wireless body area networks," Wireless Networks, vol. 26, no. 2, pp. 1265-1273, Sep. 2020, doi: 10.1007/s11276-019-02150-z.

[61] M. G. Asuti and P. I. Basarkod, "Efficiency enhancement using optimized static scheduling technique in TSCH networks," 
International Journal of Electrical and Computer Engineering (IJECE), vol. 10, no. 2, pp. 1952-1962, Apr. 2020, doi: 10.11591/ijece.v10i2.pp1952-1962.

[62] W. Zhang, X. Wang, G. Han, Y. Peng, M. Guizani, and J. Sun, "A load-adaptive fair access protocol for MAC in underwater acoustic sensor networks," Journal of Network and Computer Applications, vol. 173, Art. no. 102867, Jan. 2021, doi: 10.1016/j.jnca.2020.102867.

[63] L. de M. Guimarães and J. L. Bordim, "A full-duplex MAC technique to improve spectrum-efficiency on 5G mobile wireless networks," Computer Communications, vol. 166, pp. 216-225, Jan. 2021, doi: 10.1016/j.comcom.2020.11.020.

[64] A. A. Almohammedi, N. K. Noordin, A. Sali, F. Hashim, W. A. Jabbar, and S. Saeed, "Modeling and analysis of IEEE 1609.4 MAC in the presence of error-prone channels," International Journal of Electrical and Computer Engineering (IJECE), vol. 9, no. 5, pp. 3531-3541, Oct. 2019, doi: 10.11591/ijece.v9i5.pp3531-3541.

[65] Y. J. Lin and S. S. Tzeng, "A framed slotted aloha-based mac for eliminating vain wireless power transfer in wireless powered iot networks," Electronics (Switzerland), vol. 10, no. 1, pp. 1-19, Dec. 2021, doi: 10.3390/electronics10010009.

[66] J. Lei, J. Tao, J. Huang, and Y. Xia, "A differentiated reservation MAC protocol for achieving fairness and efficiency in multi-rate IEEE 802.11 WLANs,” IEEE Access, vol. 7, pp. 12133-12145, 2019, doi: 10.1109/ACCESS.2019.2892760.

[67] J. K. Kim, W. J. Lee, C. B. Chae, and J. H. Kim, "Performance analysis of fair medium access control protocol for asymmetric full duplex in WLAN," IEEE Access, vol. 8, pp. 140546-140557, 2020, doi: 10.1109/ACCESS.2020.3012175.

[68] H. Ahn and Y. J. Suh, "Full-duplex MAC protocol using buffer status reports during unused uplink periods in WLAN," Ad Hoc Networks, vol. 94, Art. no. 101950, Nov. 2019, doi: 10.1016/j.adhoc.2019.101950

[69] D. O. Akande and M. F. M. Salleh, "A multi-objective target-oriented cooperative MAC protocol for wireless Ad-hoc networks with energy harvesting," IEEE Access, vol. 8, pp. 25310-25325, 2020, doi: 10.1109/ACCESS.2020.2970721.

[70] J. Lei, Y. Wang, and H. Yun, "Decoupling-based channel access mechanism for improving throughput and fairness in dense multirate WLANs," Future Internet, vol. 12, no. 1, Art. no. 3, Dec. 2020, doi: 10.3390/fi12010003.

[71] Y. Gao and S. Roy, "Achieving proportional fairness for LTE-LAA and Wi-Fi coexistence in unlicensed spectrum," IEEE Transactions on Wireless Communications, vol. 19, no. 5, pp. 3390-3404, May 2020, doi: 10.1109/TWC.2020.2973145.

[72] A. Li and G. Han, "A fairness-based MAC protocol for 5G cognitive radio Ad Hoc networks," Journal of Network and Computer Applications, vol. 111, pp. 28-34, Jun. 2018, doi: 10.1016/j.jnca.2018.03.019.

[73] S. Poudel and S. Moh, "Energy-efficient and fast mac protocol in uav-aided wireless sensor networks for time-critical applications," Sensors (Switzerland), vol. 20, no. 9, Art. no. 2635, May 2020, doi: 10.3390/s20092635.

[74] P. H. Isolani, D. J. Kulenkamp, J. M. Marquez-Barja, L. Z. Granville, S. Latré, and V. R. Syrotiuk, "Support for 5G mission-critical applications in software-defined ieee 802.11 networks," Sensors (Switzerland), vol. 21, no. 3, pp. 1-36, Jan. 2021, doi: $10.3390 / \mathrm{s} 21030693$

[75] Y. Yu, S. C. Liew, and T. Wang, "Non-uniform time-step deep Q-Network for carrier-sense multiple access in heterogeneous wireless networks," IEEE Transactions on Mobile Computing, vol. 20, no. 9, pp. 2848-2861, Sep. 2021, doi: 10.1109/TMC.2020.2990399.

[76] F. Solt et al., "Energy efficient heartbeat-based MAC protocol for WBAN employing body coupled communication," IEEE Access, vol. 8, pp. 182966-182983, 2020, doi: 10.1109/ACCESS.2020.3028800.

[77] S. Sarang, G. M. Stojanović, S. Stankovski, Ž. Trpovski, and M. Drieberg, "Energy-efficient asynchronous QoS MAC protocol for wireless sensor networks," Wireless Communications and Mobile Computing, vol. 2020, pp. 1-13, Sep. 2020, doi: $10.1155 / 2020 / 8860371$.

[78] R. Ali, A. Nauman, Y. Bin Zikria, B. S. Kim, and S. W. Kim, "Performance optimization of QoS-supported dense WLANs using machine-learning-enabled enhanced distributed channel access (MEDCA) mechanism," Neural Computing and Applications, vol. 32, no. 17, pp. 13107-13115, Aug. 2020, doi: 10.1007/s00521-019-04416-1.

[79] V. Drozdova and R. Akhpashev, "The usage of load intensity balance approach for 5G MAC protocol chatacteristics investigation," in Proceedings - 2020 Ural Symposium on Biomedical Engineering, Radioelectronics and Information Technology, USBEREIT 2020, May 2020, pp. 292-294, doi: 10.1109/USBEREIT48449.2020.9117725.

[80] C. Lu, B. Wu, and T. Ye, "A novel qos-aware a-mpdu aggregation scheduler for unsaturated ieee802.11n/ac wlans," Electronics (Switzerland), vol. 9, no. 8, pp. 1-20, Jul. 2020, doi: 10.3390/electronics9081203.

[81] Z. K. Farej and M. M. Jasim, "Performance evaluation of the IEEE 802.11n random topology WLAN with QoS application," International Journal of Electrical and Computer Engineering (IJECE), vol. 10, no. 2, pp. 1924-1934, Apr. 2020, doi: 10.11591/ijece.v10i2.pp1924-1934.

[82] S. Nosheen and J. Y. Khan, "An adaptive qos based video packet transmission technique for IEEE802.11ac WLAN," in IEEE Vehicular Technology Conference, Apr. 2019, vol. 2019-April, doi: 10.1109/VTCSpring.2019.8746578.

[83] W. Rehan, S. Fischer, M. Rehan, Y. Mawad, and S. Saleem, "QCM2R: A QoS-aware cross-layered multichannel multisink routing protocol for stream based wireless sensor networks," Journal of Network and Computer Applications, vol. 156, Art. no. 102552, Apr. 2020, doi: 10.1016/j.jnca.2020.102552.

[84] M. Beshley, N. Kryvinska, M. Seliuchenko, H. Beshley, E. M. Shakshuki, and A. U. H. Yasar, "End-to-End QoS 'smart queue' management algorithms and traffic prioritization mechanisms for narrow-band internet of things services in $4 \mathrm{G} / 5 \mathrm{G}$ networks," Sensors (Switzerland), vol. 20, no. 8, Art. no. 2324, Apr. 2020, doi: 10.3390/s20082324.

[85] A. H. Sodhro, A. S. Malokani, G. H. Sodhro, M. Muzammal, and L. Zongwei, "An adaptive QoS computation for medical data processing in intelligent healthcare applications," Neural Computing and Applications, vol. 32, no. 3, pp. 723-734, Jan. 2020, doi: 10.1007/s00521-018-3931-1.

[86] V. Mishra, R. Upadhyay, U. R. Bhatt, and A. Kumar, "DEC TDMA: A delay controlled and energy efficient clustered TDMA mechanism for FiWi access network," Optik, vol. 225, Art. no. 164921, Jan. 2021, doi: 10.1016/j.ijleo.2020.164921.

[87] M. Hamiane and M. J. Ahmed, "Improvement of crankshaft MAC protocol for wireless sensor networks: A simulation study," International Journal of Electrical and Computer Engineering (IJECE), vol. 9, no. 3, pp. 1944-1956, Jun. 2019, doi: 10.11591/ijece.v9i3.pp1944-1956.

[88] S. Diakite, F. M. Rizo, and S. Y. M. Bandiri, "FD MAC protocol design for Co-existing WLANs in 5G cellular networks," Journal of Microwaves, Optoelectronics and Electromagnetic Applications, vol. 20, no. 1, pp. 30-59, Mar. 2021, doi: 10.1590/217910742021 V20I1915.

[89] Y. N. R. Li, M. Chen, J. Xu, L. Tian, and K. Huang, "Power saving techniques for 5G and beyond," IEEE Access, vol. 8, pp. 108675-108690, 2020, doi: 10.1109/ACCESS.2020.3001180.

[90] V. A. Reddy, G. L. Stuber, S. Al-Dharrab, W. Mesbah, and A. H. Muqaibel, "A wireless geophone network architecture using IEEE 802.11af with power saving schemes," IEEE Transactions on Wireless Communications, vol. 18, no. 12, pp. 5967-5982, Dec. 2019, doi: 10.1109/TWC.2019.2940944. 
[91] I. G. Lee, K. Go, and J. H. Lee, "Battery draining attack and defense against power saving wireless lan devices," Sensors (Switzerland), vol. 20, no. 7, Art. no. 2043, Apr. 2020, doi: 10.3390/s20072043.

[92] U. Sangeetha and A. V Babu, "Fair and efficient resource allocation in IEEE 802.11ah WLAN with heterogeneous data rates," Computer Communications, vol. 151, pp. 154-164, Feb. 2020, doi: 10.1016/j.comcom.2019.12.043.

[93] M. Dryjanski and A. Kliks, "A hierarchical and modular radio resource management architecture for 5G and beyond," IEEE Communications Magazine, vol. 58, no. 7, pp. 28-34, Jul. 2020, doi: 10.1109/MCOM.001.1900796.

[94] T. Praveena and G. S. Nagaraja, "Social-sine cosine algorithm-based cross layer resource allocation in wireless network," International Journal of Electrical and Computer Engineering (IJECE), vol. 11, no. 1, pp. 458-470, Feb. 2021, doi: 10.11591/ijece.v11i1.pp458-470.

[95] A. H. Sodhro et al., "Towards 5G-enabled self adaptive green and reliable communication in intelligent transportation system," IEEE Transactions on Intelligent Transportation Systems, vol. 22, no. 8, pp. 5223-5231, Aug. 2021, doi: 10.1109/TITS.2020.3019227.

[96] X. Huang, L. He, H. Xinyu, and L. He, "Playback experience driven cross layer optimisation of APP, transport and MAC layer for video clients over long-term evolution system," IET Communications, vol. 14, no. 13, pp. 2176-2188, Aug. 2020, doi: 10.1049/ietcom.2019.0645.

[97] X. Tao, C. Jiang, J. Liu, A. Xiao, Y. Qian, and J. Lu, "QoE driven resource allocation in next generation wireless networks," IEEE Wireless Communications, vol. 26, no. 2, pp. 78-85, Apr. 2019, doi: 10.1109/MWC.2018.1800022.

[98] G. Z. Islam and M. A. Kashem, "Efficient resource allocation in the IEEE 802.11ax network leveraging OFDMA technology," Journal of King Saud University - Computer and Information Sciences, Oct. 2020, doi: 10.1016/j.jksuci.2020.10.019.

[99] S. Q. Jabbar, D. J. Kadhim, and Y. Li, "Developing a video buffer framework for video streaming in cellular networks," Wireless Communications and Mobile Computing, vol. 2018, pp. 1-13, Jun. 2018, doi: 10.1155/2018/6584845. 